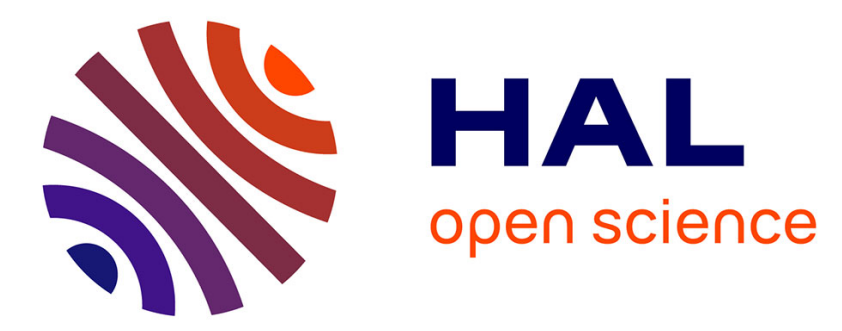

\title{
Deep-UV lithography of nanocomposite thin films into magnetooptical gratings with submicron periodicity
}

Clémentine Bidaud, Dominique Berling, Emilie Gamet, D. Jamon, Loïc Vidal, Sophie Neveu, O. Soppera, Francois Royer

\section{To cite this version:}

Clémentine Bidaud, Dominique Berling, Emilie Gamet, D. Jamon, Loïc Vidal, et al.. Deep-UV lithography of nanocomposite thin films into magnetooptical gratings with submicron periodicity. ChemPhotoChem, 2020, 10.1002/cptc.202000048 . ujm-02899691

\section{HAL Id: ujm-02899691}

\section{https://hal-ujm.archives-ouvertes.fr/ujm-02899691}

Submitted on 17 Nov 2020

HAL is a multi-disciplinary open access archive for the deposit and dissemination of scientific research documents, whether they are published or not. The documents may come from teaching and research institutions in France or abroad, or from public or private research centers.
L'archive ouverte pluridisciplinaire HAL, est destinée au dépôt et à la diffusion de documents scientifiques de niveau recherche, publiés ou non, émanant des établissements d'enseignement et de recherche français ou étrangers, des laboratoires publics ou privés. 


\section{ChemPhotoChem}

\section{Fhemistry Europe}

European Chemical Societies Publishing

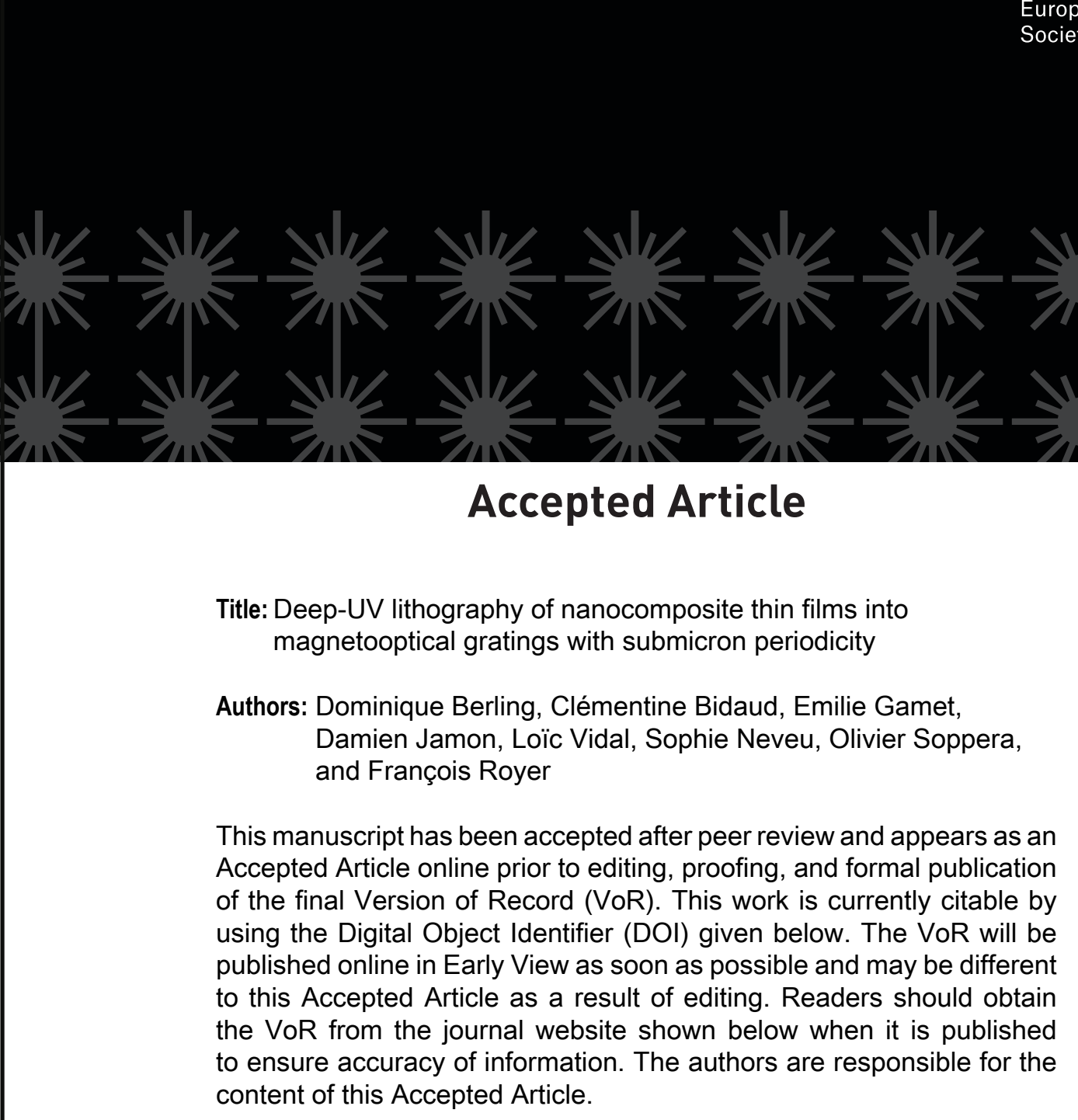

To be cited as: ChemPhotoChem 10.1002/cptc.202000048

Link to VoR: https://doi.org/10.1002/cptc.202000048 


\section{WILEY-VCH}

DOI: $10.1002 /$ cptc.202000048R1

Article type: Full Paper

Deep-UV lithography of nanocomposite thin films into magnetooptical gratings with submicron periodicity

Clémentine Bidaud ${ }^{1,2}$, Emilie Gamet ${ }^{3}$, Damien Jamon ${ }^{3}$, Lö̈c Vidal ${ }^{1,2}$, Sophie Neveu ${ }^{4}$, Olivier Soppera $^{1,2}$, François Royer ${ }^{3}$, Dominique Berling ${ }^{1,2 *}$

1. Université de Haute-Alsace, CNRS, IS2M UMR 7361, F-68100 Mulhouse, France

2. Université de Strasbourg, France

3. Université de Lyon, CNRS, UMR 5516, Institut d'Optique Graduate School, Laboratoire Hubert Curien, Université Jean-Monnet, 18 rue Pr. Lauras, F-42000 Saint-Etienne, France 4. Sorbonne Université, CNRS, Laboratoire de Physicochimie des Electrolytes et Nanosystèmes Interfaciaux, PHENIX, F-75005 Paris, France

Keywords: magneto-optics, nanocomposites, Faraday effect, micro nanopatterning, solution processing

Abstract: Incompatibility of conventional magneto-optical (MO) materials with photonicsystem fabrication processes constitutes a major technological roadblock limiting their integration into photonic devices, especially for micro- or nano-patterned films.

This paper proposes a photolithography approach toward MO micro- and nano-patterns, based on hybrid photosensitive matrices doped with magnetic nanoparticles to concentrations as high as $20 \%$ vol. The as prepared and characterized nanocomposite thin films exhibit excellent MO properties: a high Faraday rotation $(5300 \% \mathrm{~cm})$, comparable with the best existing materials that nevertheless require much more involved processes.

The photosensitive matrices allow patterned gratings with a wide range of periods $(100 \mu \mathrm{m}$ to $500 \mathrm{~nm}$ ), using a simple procedure at room temperature. The fabricated gratings exhibit welldefined regular structures and resolutions suitable for optical applications, including those at telecommunications wavelengths.

Finally, direct applications of the proposed material and its associated process are demonstrated on two examples: 1) a magneto-optical grating in free-space and 2) in combination with a waveguide. The obtained optical and MO results are consistent with simulations. 


\section{WILEY-VCH}

\section{Introduction}

Magneto-optical (MO) materials modify the light polarization in response to an external magnetic field. This property is known as the Faraday or Kerr effects, ${ }^{[1]}$ respectively, for reflection and transmission modes. Since their discovery, these effects have been used in various applications, such as data storage, ${ }^{[2]}$ three-dimensional (3D) imaging, magnonics, ${ }^{[3,4]}$ and photonics. ${ }^{[5]}$

Such properties require very specific conditions from the material point of view, especially for thin film elaboration or integration on photonic platforms. The growth of thin-film MO materials has been extensively studied using physical vapor deposition (PVD) techniques, such as sputtering, ${ }^{[6,7]}$ pulse laser deposition, ${ }^{[8]}$ and molecular beam epitaxy. ${ }^{[9,10]}$ The main drawback of these techniques is that they require sophisticated and expensive vacuum-based equipment. On the other side, efficient and low-cost chemical methods based on solutions have been proposed to simplify the preparation of thin films, ${ }^{[11]}$ including methods such as hydrothermal processing, ${ }^{[12]}$ chemical solution deposition, ${ }^{[13,14]}$ and sol-gel chemistry. ${ }^{[15]}$ In this last approach, the material deposition step is greatly simplified, and straightforward techniques, such as spin-coating or dip-coating, can be used. However, for all of the above methods, high-temperature annealing is always required as a post-treatment step, which limits the usage of MO materials in complex optical devices. Moreover, some very interesting MO materials can hardly be integrated as thin films. As an example, yttrium iron garnet doped with cerium (Ce-YIG), the most efficient MO material reported for applications at $3^{\text {rd }}$ telecom wavelength $(1.5 \mu \mathrm{m})$, requires a thermal treatment at $700{ }^{\circ} \mathrm{C}$ and should be epitaxially grown on a particular substrate, namely gadolinium gallium garnet (GGG). Under such very specific conditions, Ce-YIG exhibited the Faraday rotation (FR) at $3300 \%$, associated with the absorption of $5.8 \mathrm{~dB} / \mathrm{cm}^{[16]}$ However, when this material is integrated on conventional substrates (such as $\mathrm{Si}$ and glass), its lattice mismatch and high-temperature annealing significantly degrade its structure, consequently negatively affecting the MO properties. This 


\section{WILEY-VCH}

incompatibility between MO materials and conventional fabrication processes of photonic systems is a major technological roadblock limiting the integration of MO in photonic devices, such as integrated optical isolators. ${ }^{[17]}$

An additional current challenge in this field is providing an easy convenient process for the fabrication of micro- or nanostructured MO thin films. Indeed, micro- or nanostructures are needed for enhancing the light-matter interactions while limiting absorption-associated losses. This can be achieved via resonance phenomena such as distributed Bragg reflectors for integrated devices or resonant waveguide gratings for free-space applications. Thus, absorption losses can be compensated.

Micro- and nano-patterning is mostly achieved by classical photolithography processes, but controlled etching of such materials on the submicron scale is very difficult. ${ }^{[17]}$ For this reason, technical solutions allowing the direct writing of MO materials are of major interest and highly expected. In this context, the nanocomposite route presents specific advantages. This strategy consists of incorporating magnetic nanoparticles (MNPs) in a photopatternable host matrix. The incorporated nanoparticles (NPs) confer the MO properties while the host matrix is chosen for its capacity of being directly written in order to generate the desired micro- and nano-patterns.

Many MNP synthesis methods have been developed during the last decades. Maghemite ( $\gamma$ $\left.\mathrm{Fe}_{2} \mathrm{O}_{3}\right),{ }^{[18]}$ cobalt ferrite $\left(\mathrm{CoFe}_{2} \mathrm{O}_{4}\right),{ }^{[19]}$ and mixed ferrite NPs $\left(\mathrm{MFe}_{2} \mathrm{O}_{4}\right)^{[20]}$ are synthesized by several processes, such as co-precipitation, ${ }^{[21]}$ hydrothermal processing, ${ }^{[22]}$ and hightemperature decomposition. ${ }^{[23]}$

Such NPs can be integrated in host matrices. Silicon-based sol-gel matrices have been successfully developed to host cobalt ferrite NPs, for applications such as polarization converters integrated on glass. ${ }^{[6]}$ Compared with the PVD of MO materials, this nanocomposite route allows the integration of MO elements at moderate temperatures. Indeed, the temperature required to crosslink the matrix can be as low as $100{ }^{\circ} \mathrm{C}$. In addition to the 


\section{WILEY-VCH}

need for a thermal post-treatment (although it occurs at moderate temperatures), another restriction is the limited patterning method based on templating (nanoimprints, 3D structuring with sacrificial templates ${ }^{[24,25]}$, which is the only one compatible with silicon-based sol-gel materials.

Incorporation of an organic component in a hybrid organic-inorganic sol-gel matrix opens the possibility to achieve patterning at room temperature (RT). The final material properties are mostly determined by the inorganic component, while the organic component contributes photopatternable properties. Among different approaches, 3methacryloxypropyltrimethoxylsilane (MAPTMS) has been widely used for optical applications, owing to its excellent patterning potential and interesting optical properties (refractive index and low losses at the telecom wavelength). ${ }^{[26,27]}$

In this work, we report the preparation of a novel photopatternable hybrid nanocomposite with high-performance MO properties. In addition, the reported material can be patterned by deep ultraviolet (DUV) light $(193 \mathrm{~nm})$ in the sub-micrometric resolution. Interferometric DUV irradiation has been chosen as a patterning method since it allows patterning relatively wide areas $\left(\sim 1 \mathrm{~cm}^{2}\right)$ in a single exposure of a few minutes.

The remainder of this paper is divided into three parts. First, the material preparation and the characterization of the thin films with MO properties are described. Then, the potential for sub-micrometric-scale photopatterning is discussed and demonstrated. Finally, simulations and experimental measurements of structured samples demonstrate the potential of this material for MO components to be used in integrated devices.

\section{Formulation and photochemistry}

Figure 1 presents the photostructured MO material preparation modus operandi: from the formulation of a sol-gel hybrid magnetic nanocomposite to its laser photopatterning by DUV. The hybrid matrix was obtained by following a classical sol-gel route. On one side, 3- 


\section{WILEY-VCH}

methacryloxypropyltrimethoxylsilane (MAPTMS) is pre-hydrolyzed with hydrochloric acid. On the other side, titanium isopropoxide (TTIP) is reacted with an excess of methacrylic acid (MAA). The complex formed by the reaction of the Ti precursor with the MAA is known to be stable with respect to hydrolysis, which avoids uncontrolled precipitation of the $\mathrm{Ti}$ precursor. These solutions are then mixed in different proportions, for tuning the molar ratio Si:Ti from 3:1 to 20:1 (Figure 1a). The addition of water is important to ensure the hydrolysis-condensation reactions of the silicon-based precursor, and for obtaining highoptical-quality smooth films by spin-coating (Figure 1d).

This organic-inorganic liquid matrix is then doped with a $\mathrm{CoFe}_{2} \mathrm{O}_{4} \mathrm{NP}$ ferrofluid. MNPs (average size, $8 \mathrm{~nm}$ ) have been synthesized following the co-precipitation route ${ }^{[28]}$ (Figure 1b). Up to $20 \%$ vol MNPs can be homogeneously dispersed in the liquid material. The as formulated solutions showed excellent stability (equivalent properties after more than one year, even for the highest MNP doping up to 20\%vol (Figure 1c).

The bottom panel in Figure 1 outlines the thin film preparation process by spin-coating the composite solution (Figure 1d) and its photopatterning by the DUV lithography (Figure 1e). The material behaves as a negative-tone photoresist under irradiation (Figure 1f).

The wavelength of $193 \mathrm{~nm}$ was chosen to avoid introducing photoinitiating molecules into the hybrid MAPTMS-TTIP based formulation. The formulation has thus been adapted from previous studies, to deal with these conditions. ${ }^{[29]}$ Indeed, photocrosslinking is achieved by radical photopolymerization of the MAPTMS methacrylate functions. Under the UV irradiation, a photoinitiator is needed to trigger the free-radical photopolymerization of MAPTMS. ${ }^{[27]}$ We propose here an original mechanism based on the DUV-induced decomposition of the $\mathrm{Ti}$ complexes. Ti complexes have already shown their potential to produce radicals under DUV irradiation. ${ }^{[30,31]}$ This mechanism is supported by the reaction kinetics obtained by investigating the photocrosslinking under the DUV irradiation by Fourier transform infrared (FT-IR) spectroscopy, and is shown in Figures $2 \mathbf{a}$ and $2 \mathbf{b}$. 


\section{WILEY-VCH}

The polymerization of the methacrylate functions was characterized by plotting the temporal evolution of the $1638 \mathrm{~cm}^{-1}$ band versus the irradiation time. A very good final conversion ratio was obtained, in the range of $80-100 \%$, depending on the titanium content (Figure 2a) and on the concentration of NPs (Figure 2b). The material can be efficiently cross-linked by photoirradiation, within a few minutes.

There is a clear correlation between the Ti complex loading in the thin film and the rate of polymerization. Increasing the Ti complex content allows reaching the maximal conversion after $100 \mathrm{~s}$ of irradiation (at $70 \mathrm{~mW} / \mathrm{cm}^{2}$ ). This is consistent with a free-radical polymerization initiated by the decomposition of the Ti-MAA complex, as reported by Soppera et al. ${ }^{[29]}$ It is consequently more interesting to use a material containing more $\mathrm{Ti}$, to allow faster and efficient photostructuration. Indeed, using the 3:1 molar ratio is better from the kinetic polymerization viewpoint, but it was observed that increasing the Ti content reduces the longterm solution stability. Thus, as a compromise between those two requirements (kinetic polymerization vs. solution stability), the Si:Ti molar ratio was set to $6: 1$ in the subsequent studies.

The impact of MNPs on the photopolymerization is another important consideration. UVvisible absorption spectra (Figure 2c) show that in the DUV range, the ferrofluid absorption is high. The MNPs inserted into the matrix may thus significantly decrease the efficiency of the photopolymerization by absorption. As shown in Figure $\mathbf{2 b}$, the MNPs do not significantly affect the photopolymerization kinetics, for concentrations below $2 \%$ vol. From $5 \%$ vol to $20 \% \mathrm{vol}$, a drop in the polymerization rate is observed, resulting in longer irradiation times for crosslinking nanocomposite films. However, even for the highest MNP content, efficient crosslinking was achieved (qualitatively demonstrated by the resistance to etching by solvents), with the final conversion above $70 \%$. In addition, no significant degradation of the mechanical properties of the thin film with the lowest conversion ratio has been observed. 


\section{WILEY-VCH}

This demonstrates that the nanocomposite material retains its properties even for very high MNP contents.

Interestingly, we observed no significant shrinkage of the material in response to the irradiation: the thickness decrease did not exceed 3-5\% of the initial film thickness, which could be attributed to the loss of organic compounds, such as carbon dioxide, under the DUV irradiation. This is important for ensuring final optical properties.

The final nanocomposite material was further characterized using transmission electron microscopy (TEM). Figure 2e shows a section of the cured material with the $10 \%$ vol MNP content. In this case, MNPs tended to form small aggregates in the nanometer range (Figure 2e), but the MNPs distribution in the matrix was quite homogeneous, yielding a transparent material, without significant scattering. The chemical maps obtained by the energy-dispersive X-ray (EDX) analysis (Figure 2f) indicate a uniform Si and Ti repartition throughout the entire sample, while Fe and Co are, as expected, detected only in the MNPs.

The magneto-optical properties of the fabricated DUV-photopolymerized nanocomposite thin films were monitored as well. The hysteresis loops obtained by measuring the Faraday rotation in response to the applied field at $781 \mathrm{~nm}$ are shown in Figure 2d. The Faraday rotation, proportional to the material magnetization, is the rotation of the light polarization direction that is observed when light rays pass through a sample in a magnetic field. The absolute value of the Faraday rotation measured at saturation scales linearly with the NP content over the entire NP doping range. Independent of the concentration, hysteresis loops are open, with a remanent effect and a coercive field. This observation suggests that, if MNPs exhibit superparamagnetic behavior when they are free to move in the ferrofluid, this behavior is lost once the particles are inserted in the matrix. In the polymerized material, the MNPs are indeed blocked in rotation, and a hysteresis loop appears owing to the strong magnetocrystalline anisotropy of cobalt ferrite. ${ }^{[32]}$ 


\section{WILEY-VCH}

The previously demonstrated crosslinking reaction induced by the DUV irradiation could be used for photopatterning. As for negative tone resists, the irradiated area becomes insoluble upon the irradiation and the non-irradiated parts can be eliminated by immersing the sample in a suitable solvent. The rationale of this approach was to obtain structured thin films with magneto-optical properties immediately after photopatterning. The formulation was deposited by spin-coating. Film thicknesses, measured by ellipsometry, were in the $70-500 \mathrm{~nm}$ range.

Photopatterning was achieved by three techniques that allow to generate regular patterns with different periods: i) proximity mask lithography with a chromium mask was used for generating 1.6- $\mu \mathrm{m}$-period (line width, $800 \mathrm{~nm}$ ) and interference lithography ii) with one and iii) two phase masks used for generating, respectively, 500-nm-width and 600-nm-width periodic lines. Interestingly, these three setups are complementary in terms of pattern dimension and light pattern profiles. Indeed, a mask lithography configuration generates a binary light pattern, but the smallest patterned line period is limited to $1.6 \mu \mathrm{m}$. On the other hand, interference lithography is efficient for generating submicron patterns and, in this case, the light pattern is sinusoidal. The energy required to obtain the structures, regardless of the setup, is in the $100-300 \mathrm{~mJ}$ range, corresponding to the $1.5-\mathrm{mW}$-power DUV in a few minutes of laser irradiation. After the irradiation, the samples were developed in cyclohexanone and dried under the $\mathrm{N}_{2}$ flux to reveal the structures.

In Figure 3a, the atomic force microscopy (AFM) topography images show the typical structures obtained for the both setups. Regular and well-defined structures over the area of a few squared millimeters were obtained. Regardless of the period, the higher the concentration of MNPs, the more aggregates appeared at the surface, as evidenced by the AFM images (Figure 3). There is a clear difference between the well-defined regular smooth structures observed for the low vs. high doping regimes.

The effect of the irradiation energy on the height of the structures was investigated using AFM. The line height was determined using the height-height autocorrelation function 


\section{WILEY-VCH}

applied to the topographical images. This method yielded the average height for a large (typically, $10 \times 10 \mu \mathrm{m}^{2}$ ) surface. As shown in Figure 3b, there is an optimal energy value for which the structures' height is maximal. For the lowest MNP doping ( $<5 \%$ vol), the maximal grating height is $\sim 40 \%$ of the total thickness of the initial film. For the highest MNP content (range, 5-20\%vol) this value decreases to $20-25 \%$. This directly follows owing to the NP doping and absorption.

For this optimal energy value, the material is not sufficiently polymerized, and is totally removed during development. For higher energy values, the material starts polymerizing between the lines, and the structures' height decreases. This behavior is independent of the patterning setup. The final structure is thus composed of a continuous reticulated thin film at the substrate interface on which the periodical structure is created.

The energy required for modulations increases with increasing the MNP content: for low concentrations, $100 \mathrm{~mJ}$ (i.e., less than one minute of irradiation) of energy are required, but the required energy increases up to $300 \mathrm{~mJ}$ for higher concentrations. It is still interesting to note that this range of energies corresponds to the irradiation time of several minutes. The fabrication of such systems in one step and at RT is consequently very fast.

\section{Magneto-optical properties and devices}

For exemplification, two grating structures were fabricated; the first one addresses free space optics while the second one addresses integrated optics.

A possibility of the enhancement of the MO effects by one free-space dielectric grating has been theoretically demonstrated. ${ }^{[33,34]}$.The first experimental results were obtained for a highdielectric-index impregnated grating template, ${ }^{[33]}$ with an MNP-doped mineral sol-gel. The possibility of photopatterning a composite material, demonstrated above, suggests the possibility to fabricate MO gratings without high-index templates, which would reduce the number of fabrication steps as well as the process cost. Thus, a grating on a Pyrex substrate 


\section{WILEY-VCH}

was realized, with the following parameters (Figure 4a): 13\%vol NP doped composite, leading to the refractive index of $1.6+\mathrm{i} 0.0183$ at the $1.55 \mu \mathrm{m}$ wavelength, $1.6 \mu \mathrm{m}$ period, 110 nm depth, and a 430-nm-thick sub-layer. The corresponding simulated structure took into account the dispersion of the refractive index of the photostructurable nanocomposite. MO modelling was performed using a custom RCWA code, ${ }^{[33]}$ taking into account the entire permittivity tensor. ${ }^{[35]}$ The Fourier modal method (FMM) or RCWA is the most suitable numerical method for analyzing the interactions of electromagnetic waves with periodic diffractive structures.

The grating presented here is not optimized for the Faraday rotation enhancement, as the reachable depth of modulation, the line-space ratio as well as the available periods are quite limited. Thus, the efficiency of the TE transmission dip, measured at $1512 \mathrm{~nm}$, is not important, but is consistent with the simulation results (Figure 4b). Nevertheless, the simulation results (Figure 4c) suggest the MO activity and a spectral modification of the Faraday rotation compared with that of a 540-nm-thick thin film. The Faraday rotation was also measured for the tested device (Figure 4d). Even if it is weak, the measurements evidence the spectral modification induced by the grating on the Faraday effect in the area of the TE resonance. The slight offset in wavelength $(\sim 20 \mathrm{~nm})$ observed between the simulation and the measurements is due to a small error on the angle of incidence on the experimental setup. These modifications are in a good agreement with the simulation results, not only regarding the spectral behavior, but also with respect to the magnitude of the effects (Figure 4d).

The second structure consists of a $500 \mathrm{~nm}$ periodic grating doped with NPs at $0.4 \%$ vol. Compared with the previous device, the concentration of NPs is lower, because the propagation in the waveguide is on the order of several centimeters; a concentration as high as $13 \%$ would yield too much absorption, and would preclude from detecting light at the output of such a device. The structure is composed of a homogeneous 740-nm-thick film with 36- 


\section{WILEY-VCH}

nm-high modulations atop of it. The sample is shown schematically in Figure 5a. This structure was prepared on a waveguide produced by ion exchange in glass, ${ }^{[36,37]}$ and was configured as a one-dimensional photonic crystal. Such a device is known to enhance the guided magneto-optical effect, at the edge of the Bragg photonic bandgap. ${ }^{[38]}$ The Bragg wavelength $\left(\lambda_{B}\right)$ is linked to the period $(\Lambda)$ and effective index $\left(n_{\text {eff }}\right)$ of the waveguide mode, as follows: $\lambda_{B}=2 \cdot \Lambda \cdot n_{\text {eff }}$. The effective index obtained using the beam propagation method (BPM) with Opti-BPM (Optiwave Systems Inc.) software, ${ }^{[39]}$ is on the order of 1.51 (Figure 5), yielding the required period of $500 \mathrm{~nm}$ for obtaining the Bragg effect at $\sim 1500 \mathrm{~nm}$. The image of the light beam exiting the device, shown in Figure 5b, confirms the lightguiding ability of the device. This demonstrates that such photosensitive composite approach offers the possibility of depositing and structuring MO materials on optical chips, while retaining the guiding ability (Figure 5b). Indeed, the material is directly spin-coated and irradiated onto this particular substrate, without any degradation of the wave guiding properties. Compared with the work of Levy et al., ${ }^{[38]}$ where an MO material was patterned using a focused ion beam, the currently proposed approach is especially attractive for easy fabrication of MO-based guided resonant devices with a sub-micrometric period.

\section{Conclusion}

In this work, we presented a magneto-optical nanocomposite material that was photostructured as gratings with micro- and sub-micrometrical periods, for fabrication of optical devices. The material is based on a sol-gel host matrix prepared from precursors of silicon and titanium, in which cobalt ferrite NPs were incorporated. These MNPs exhibited good MO properties, in particular a strong Faraday rotation at the wavelength of $1550 \mathrm{~nm}$. The nanocomposite could be doped by up to $20 \%$ vol with particles, which conferred to the material the FR of $5300 \% \mathrm{~cm}$. This is an excellent characteristic, compared with currently available MO materials. 


\section{WILEY-VCH}

The nanocomposite was prepared as thin films and photostructured as gratings with periods ranging from $100 \mu \mathrm{m}$ to $500 \mathrm{~nm}$. For each period, regular and well-defined structures were obtained. Owing to its formulation and structuration flexibility, this magneto-optical nanocomposite is particularly promising for optical applications.

We demonstrated the use of this structured material on a waveguide, in free-space and guidedwave configurations. The Faraday measurements for the free-space grating showed a modification of the spectral Faraday rotation. The steps of the fabrication of the grating on a waveguide are compatible with the requirements on this substrate. In both cases, the use of structured magneto-optical nanocomposites for preparing optical devices is very promising.

\section{Experimental Section}

Chemicals: 3-methacryloxypropyltrimethoxylsilane (MAPTMS), titanium isopropoxide (TTIP), methacrylic acid (MAA), hydrochloric acid (HCl), 1-propanol, and cyclohexanone were purchased from Sigma Aldrich. Deionized water was used throughout the reactions.

Formulation of the matrix: The MAPTMS was prehydrolyzed with the hydrochloric acid $(0.01 \mathrm{~mol} / \mathrm{L})$ at the molar ratio of $1.3 / 1 \mathrm{MAPTMS} / \mathrm{H}_{2} \mathrm{O}$. The solution was magnetically stirred for $1 \mathrm{~h}$, to obtain a colorless emulsion. The TTIP was added to the MAA, at the 2.2 MAA/TTIP molar ratio. The solution was stirred for $5 \mathrm{~min}$. 1-propanol was then added to the solution at the 0.9 molar ratio of MAA/1-propanol. This yellow homogeneous solution was stirred for $10 \mathrm{~min}$. The two solutions (MAPTMS and TTIP) were mixed together and water was quickly added at the 0.4 molar ratio of MAPTMS/ $\mathrm{H}_{2} \mathrm{O}$. The solution was stirred for a few minutes, to obtain a homogeneous matrix. This matrix was then doped with cobalt ferrite NPs by adding a certain volume of a ferrofluid containing the MNPs inside a given volume of the matrix. The nanocomposite solution was sonicated for 5-60 min (depending on the doping, with higher doping requiring longer times). The material was diluted with 1-propanol to be prepared as thin films by spin-coating. 


\section{WILEY-VCH}

Synthesis of $\mathrm{CoFe}_{2} \mathrm{O}_{4} \mathrm{NPs}$ : The preparation of cobalt ferrite NPs was performed by the precipitation of cobalt chloride and ferric chloride (molar ratio 1:2), based on the method of Massart and Tourinho. ${ }^{[28]}$ For stabilization in an acidic solution, cobalt ferrite NPs were protected by a ferric nitrate treatment $\left(\left[\mathrm{Fe}\left(\mathrm{NO}_{3}\right)_{3}\right]=0.3 \mathrm{M}\right)$. After this treatment, magnetic NPs were electrostatically stabilized in an acidic solution, and a stable ferrofluid was obtained with the $\mathrm{pH}$ of $\sim 2$.

Fourier transform infrared (FTIR) spectroscopy: The photopolymerization kinetics were followed by FTIR, using a ThermoFisher Scientific Nicolet 8700, coupled with a Hamamatsu high-intensity mercury-xenon lamp equipped with a light guide (Lightnincure series LC8 lamp). Si wafers (thickness, $0.25 \mathrm{~mm}$ ) were used as substrates, and the irradiance was 70 $\mathrm{mW} / \mathrm{cm}^{2}$.

UV-Vis spectroscopy: Absorption measurements were performed using a Lambda $950 \mathrm{UV} / \mathrm{Vis}$ instrument (Perkin Elmer).

Ellipsometry analysis: Measurements of the thicknesses of the thin films and their optical properties ( $\mathrm{n}$ and $\mathrm{k}$, respectively, corresponding to the optical index and extinction coefficient) were performed using ellipsometric spectroscopy. The measurements were performed using a UVISEL ellipsometer from Horiba-Jobin-Yvon (spectral range, 190-830 $\mathrm{nm})$. The acquired data were fitted using the proprietary software that was delivered with the UVISEL ellipsometer.

Transmission electron microscopy: Transmission electron microscopy (TEM) was performed using a JEOL ARM-200F microscope operating at the $200 \mathrm{keV}$ accelerating voltage. The chemical analysis was performed using a JEOL Centurio detector. The samples were prepared for observation using a Leica ultramicrotome, model EM-UC7, operating at RT. To observe the film cross-section, it was deposited on the surface of a thermanox substrate. The slide thickness was $\sim 100 \mathrm{~nm}$. 


\section{WILEY-VCH}

Nano- and micro-photostructuration: Structures were realized using a Braggstar ArF nanosecond excimer laser (coherent), emitting at $193 \mathrm{~nm}\left(0.5 \mathrm{~mW} / \mathrm{cm}^{2}\right)$. Optical setups for photopatterning are described in the main text.

Atomic force microscopy: The micro- and nanostructured films were characterized using atomic force microscopy (AFM), in the tapping mode, using a PicoPlus 5500 System model from Agilent.

Magneto-optic measurements: The magneto-optical behavior of the free-space grating was investigated using a home-made polarimetric optical bench, based on the modulation technique combined with an ellipsometric-type calibration method (see ${ }^{[40]}$ for details). This system mainly employs a xenon white light source (combined with a monochromator for selecting the wavelength), a polarizer, a photoelastic modulator, an analyzer, a detector, and a lock-in amplifier (LIA). Such an optical arrangement is suitable for analyzing the polarization state of light in terms of the first and second harmonics of the LIA. ${ }^{[41]}$ The calibration method $^{[42,43]}$ allows to measure the absolute value of the polarization rotation with the detection limit of $0,001^{\circ}$. Thus, the Faraday effect was measured as a function of the field through a hysteresis loop curve. The saturated value was then plotted as a function of the wavelength.

\section{Acknowledgements}

This work was supported by funding from Agence National pour la Recherche (project PhotoMagnet, ANR-16-CE09-0017-01) and from Material Institute Carnot Alsace (projet PHOTO-MAGNET). The authors also gratefully acknowledge Université de Haute Alsace Mulhouse for Clementine Bidaud PhD funding.

The authors thank IMEP LAHC laboratory, Grenoble, France for the waveguides supply and the BPM Optiwave simulations.

\section{Conflict of Interest}

The authors declare no conflict of interest.

Received: ((will be filled in by the editorial staff))

Revised: ((will be filled in by the editorial staff)) Published online: ((will be filled in by the editorial staff)) 


\section{WILEY-VCH}

References

[1] B. E. A. Saleh, M. C. Teich, Fundamentals of Photonics, John Wiley \& Sons, Inc. (Wiley Series in Pure and Applied Optics), 1991

[2] E. Betzig, J. K. Trautman, R. Wolfe, E. M. Gyorgy, P. L. Finn, M. H. Kryder, C. H. Chang, Appl. Phys. Lett. 1992 61, 142-144

[3] A. A. Serga, A. V. Chumak, B. Hillebrands, J. Phys. D: Appl. Phys. 2010 43, 264002

[4] V. V. Kruglyak, S. O. Demokritov, D. Grundler, J. Phys. D: Appl. Phys. 2010 43, 260301

[5] H. Amata, F. Royer, F. Choueikani, D. Jamon, F. Parsy, J.-E. Broquin, S. Neveu, J. J. Rousseau, Appl. Phys. Lett. 2011 99, 251108

[6] M. Djamal, R. Ramli, Khairurrijal, F. Haryanto, Acta Physica Polonica A 2015, 128

[7] S. Schnittger, C. Jooss, S. Sievers, J. Phys.: Conf. Ser. 2010 200, 072086

[8] R. S. Hassan, N. Viart, C. Ulhaq-Bouillet, J. L. Loison, G. Versini, J. P. Vola, O. Crégut, G. Pourroy, D. Muller, D. Chateigner, Thin Solid Films 2007, 515, 2943

[9] S. A. Chambers, R. F. C. Farrow, S. Maat, M. F. Toney, L. Folks, J. G. Catalano, T. P. Trainor, G. E. Brown, J. Magn. Magn. Mater. 2002, 246, 124

[10] Y. Suzuki, R. B. Van Dover, E. M. Gyorgy, J. M. Phillips, V. Korenivski, D. J. Werder, C. H. Chen, R. J. Cava, J. J. Krajewski, W. F. Peck, K. B. Do, Appl. Phys. Lett. 1996, 68, 714 [11] C. C. Yeh, H. W. Zan, O. Soppera, Advanced Materials 2018, 30, 24

[12] F. Cheng, C. Liao, J. Kuang, Z. Xu, C. Yan, L. Chen, H. Zhao, Z. Liu, J. Appl. Phys. $1999,85,2782$

[13] J. Buršík, M. Soroka, R. Uhrec, R. Kužel, F. Mika, S. Huber, Appl. Surf. Sci. 2016, 376, 209

[14] Y. Q. Dai, J. M. Dai, X. W. Tang, Z. F. Zi, K. J. Zhang, X. B. Zhu, J. Yang, Y. P. Sun, J. Magn. Magn. Mater. 2015, 394, 287 


\section{WILEY-VCH}

[15] X. W. Wang, Y. Q. Zhang, H. Meng, Z. J. Wang, Z. D. Zhang, J. Alloys Compd. 2011, 509,7803

[16] L. Bi, J. Hu, P. Jiang, H. S. Kim, D. H. Kim, M. C. Onbasli, G. F. Dionne, C. A. Ross, Materials 2013, 6, 5094

[17] M. C. Onbasli, L. Beran, M. Zahradník, M. Kučera, R. Antoš, J. Mistrík, G. F. Dionne, M. Veis, C. A. Ross, Scientific Reports 2016, 6, 23640

[18] A. P. Safronov, O. M. Samatov, I. S. Tyukova, E. A. Mikhnevich I. V. Beketov, J. Magn. Magn. Mater. 2016, 415, 24

[19] R. Muzzalupo, L. Tavano, C. O. Rossi, N. Picci, G. A. Ranieri, Colloids and Surfaces B: Biointerfaces 2015, 134, 273

[20] R. Messing, N. Frickel, L. Belkoura, R. Strey, H. Rahn, S. Odenbach, A. M. Schmidt, Macromolecules, 2011 44, 2990

[21] F. A. Tourinho, R. Franck, R. Massart, J. Mater. Sci. 1990, 25, 3249

[22] V. Cabuil, V. Dupuis, D. Talbot, S. Neveu, J. Magn. Magn. Mater. 2011, 323, 1238

[23] A. P. Herrera, L. Polo-Corrales, E. Chavez, J. Cabarcas-Bolivar, O. N. C. Uwakweh, C. Rinaldi, J. Magn. Magn. Mater. 2013, 328, 41

[24] T. Nakahara, J. Suzuki, Y. Hosokawa, F. Shimokawa, H. Kotera, T. Suzuki, Magnetochemistry, 2018, 4, 7

[25] M. Suter, O. Ergeneman, J. Zürcher, S. Schmid, A. Camenzind, B. J. Nelson, C. Hierold, J. Micromech. Microeng. 2011, 21, 025023

[26] S. Jeong, J. Moon, J. Non-Cryst. Solids 2005, 351, 3530

[27] H.Y. Ma, T.L. Wang, P.Y. Chang, C.H. Yang, Nanomaterials 2016, 6, 44

[28] F. Tourinho, R. Franck, R. Massart, R. Perzynski, In: P. Bothorel, E.J. Dufourc (eds) Trends in Colloid and Interface Science III. Progress in Colloid \& Polymer Science, 1989, 79, 128

[29] O. Soppera, C. Croutxé-Barghorn, C. Carré, D. Blanc, Appl. Surf. Sci. 2002, 186, 91 


\section{WILEY-VCH}

[30] V. Davy-Louis, O. Soppera, L. Jacques, C.-B. Céline, New. J. Chem. 2008, 32, 2270

[31] O. Soppera, C. Croutxé-Barghorn, D. J. Lougnot, New J. Chem. 2001, 25, 1006

[32] A. Virden, S. Wells, K. O’Grady, J. Magn. Magn. Mater. 2007, 316, e768

[33] E. Gamet, B. Varghese, I. Verrier, F. Royer, J. Phys. D: Appl. Phys. 2017, 50, 495105

[34] I. S. Maksymov, J. Hutomo, and M. Kostylev, Optics Express 2014, 22, 8720-8725

[35] L. Li, Reformulation of the Fourier modal method for surface-relief gratings made with anisotropic materials. Journal of Modern Optics 1998, 45, 1313-1334

[36] O. Bertoldi, J.-E. Broquin, G. Vitrant, V. Collomb, M. Trouillon, V. Minier, in Proc. SPIE 5451, Integrated Optics and Photonic Integrated Circuits (Eds: C. Giancarlo Righini, S. Honkanen), 2004, 182-190

[37] J. Grelin, E. Ghibaudo, J.-E. Broquin, Materials Science and Engineering B 2008, 149, 185

[38] R. Li, M. Levy, Applied Physics Letters 2005, 86, 251102

[39] Opti-BPM (Optiwave Systems Inc.) Software, https://optiwave.com/ (Website, accessed: December, 2019)

[40] E. Abou-Diwan, F. Royer, D. Jamon, R. Kekesi, S. Neveu, M.F. Blanc-Mignon, J.J. Rousseau, J. Nanosci. Nanotechnol. 2016, 6, 10160

[41] B. Drevillon, Prog. Cryst. Growth Charact. Mater. 1993, 27, 1

[42] G. Jellison, F. Modine, in Proc. SPIE 1166, Polarization Considerations for Optical Systems II (Eds: Russell A. Chipman) 1990, 231-241

[43] S. Kouko, C. Llinares, J. Phys. III France 1995, 5, 1211 


\section{WILEY-VCH}

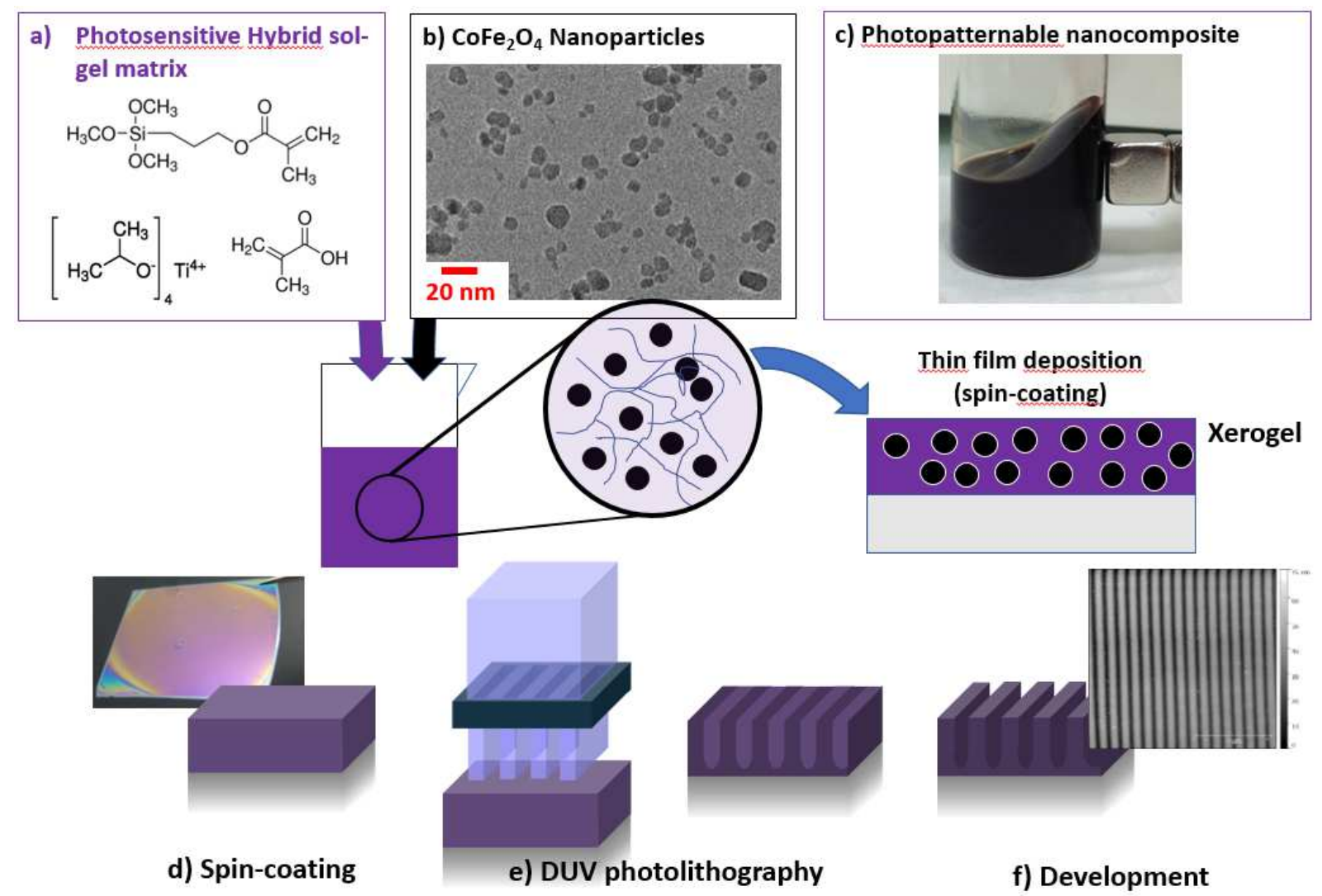

Figure 1. General summary of the preparation of the magneto-optical gratings from the nanocomposite: a) A photosensitive hybrid sol-gel matrix is obtained after hydrolysis and condensation reactions by mixing MAPTMS (pre-hydrolysis of the alkoxysilane (MAPTMS) with the hydrochloric acid) and Ti (complexation of the titanium alkoxide (TTIP) with the methacrylic acid (MAA) for obtaining metallic oxoclusters) solutions and by the addition of water; b) the matrix is mixed with MNPs of cobalt ferrite, to obtain c) a hybrid magnetooptical nanocomposite, which can be doped by up to $20 \%$ vol with NPs; d) a liquid nanocomposite is prepared as thin films by spin-coating, and is e) photostructured using lithography methods for obtaining f) gratings with micro- and submicrometer periods after devlopment. 


\section{WILEY-VCH}

a)
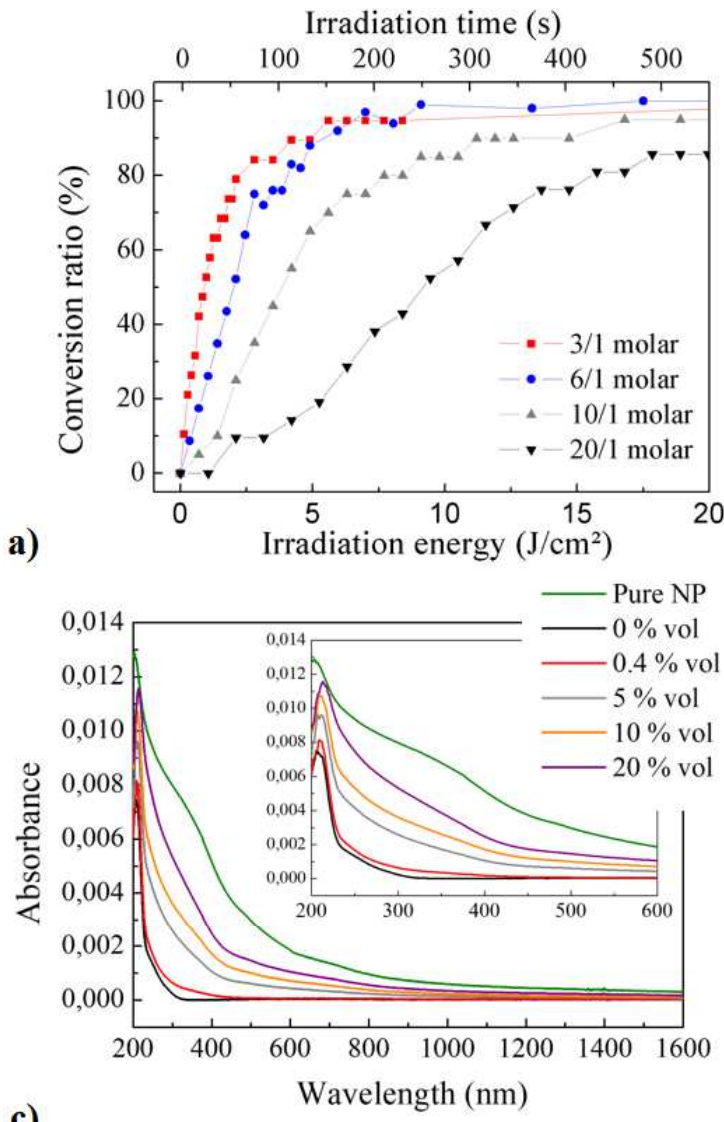

c)

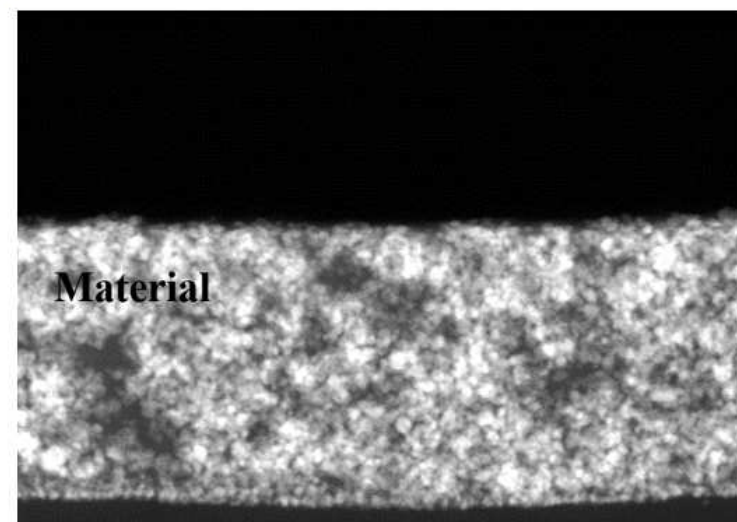

Substrate

e)

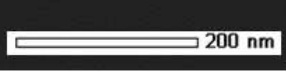

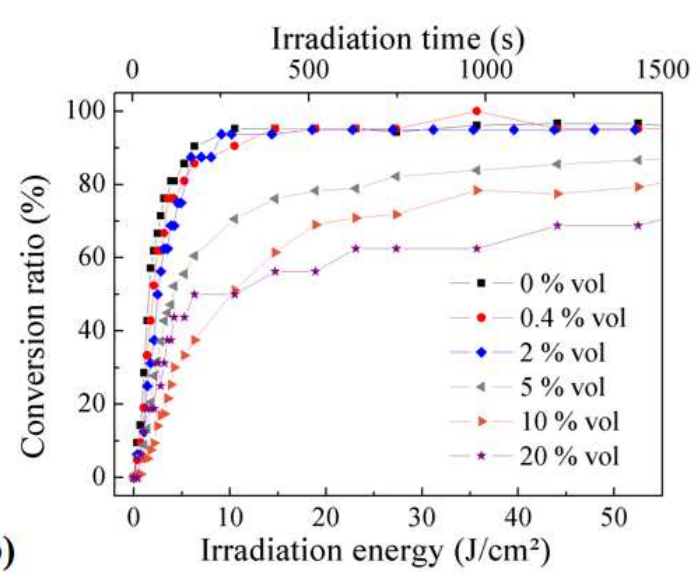

d)

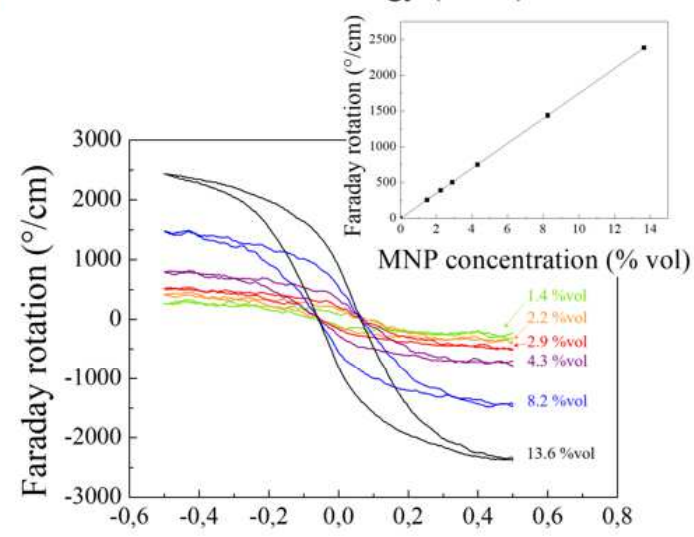

Applied magnetic field ( $\mathrm{T})$
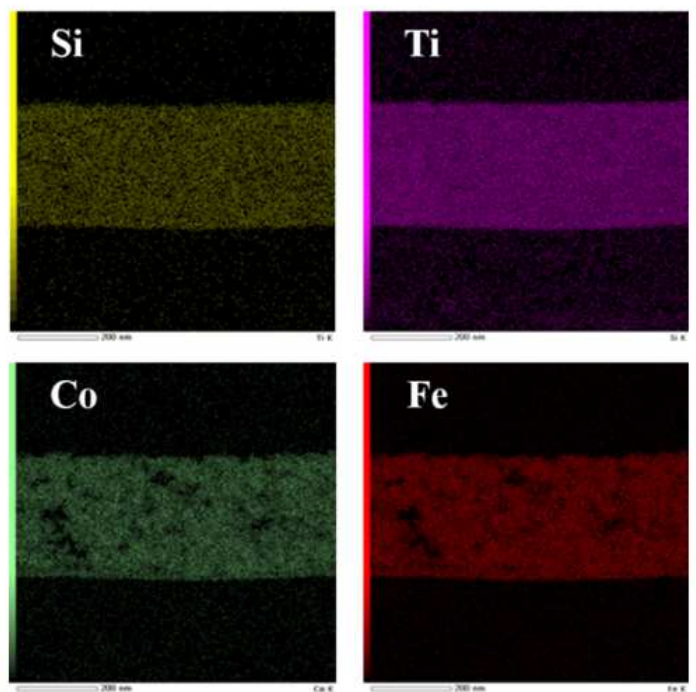

Figure 2. Photopolymerization reaction kinetics based on the monitoring of the decrease of the $\mathrm{C}=\mathrm{C}$ band at $1638 \mathrm{~cm}^{-1}$ a) for different $\mathrm{Si}$ :Ti molar ratios (films with thicknesses of $450 \pm$ $10 \mathrm{~nm}$, and with MNP doping of $0.4 \% \mathrm{vol}$ ), b) for different NP concentrations (film thickness, $450 \pm 10 \mathrm{~nm}$ ) in the $0 \%-20 \%$ vol range, with the Si:Ti molar ratio set to 6:1, c) UV-visible spectrometry absorption spectra of various NP concentration formulations $(\mathrm{Si}: \mathrm{Ti}=6: 1$ ) (an amplified view into the UV region is given in the figure inset), d) Faraday rotation $(\% \mathrm{~cm}) \mathrm{vs}$. applied field for photopolymerized thin films (film thickness, $450 \pm 10 \mathrm{~nm}$ ) for different NP contents, measured at $781 \mathrm{~nm}$, e) TEM cross-sectional view of a polymerized film doped at 10\% vol NPs and with Si:Ti = 6:1 (NPs appear bright), f) EDX analysis (chemical mapping) obtained from the previous TEM image. 


\section{WILEY-VCH}

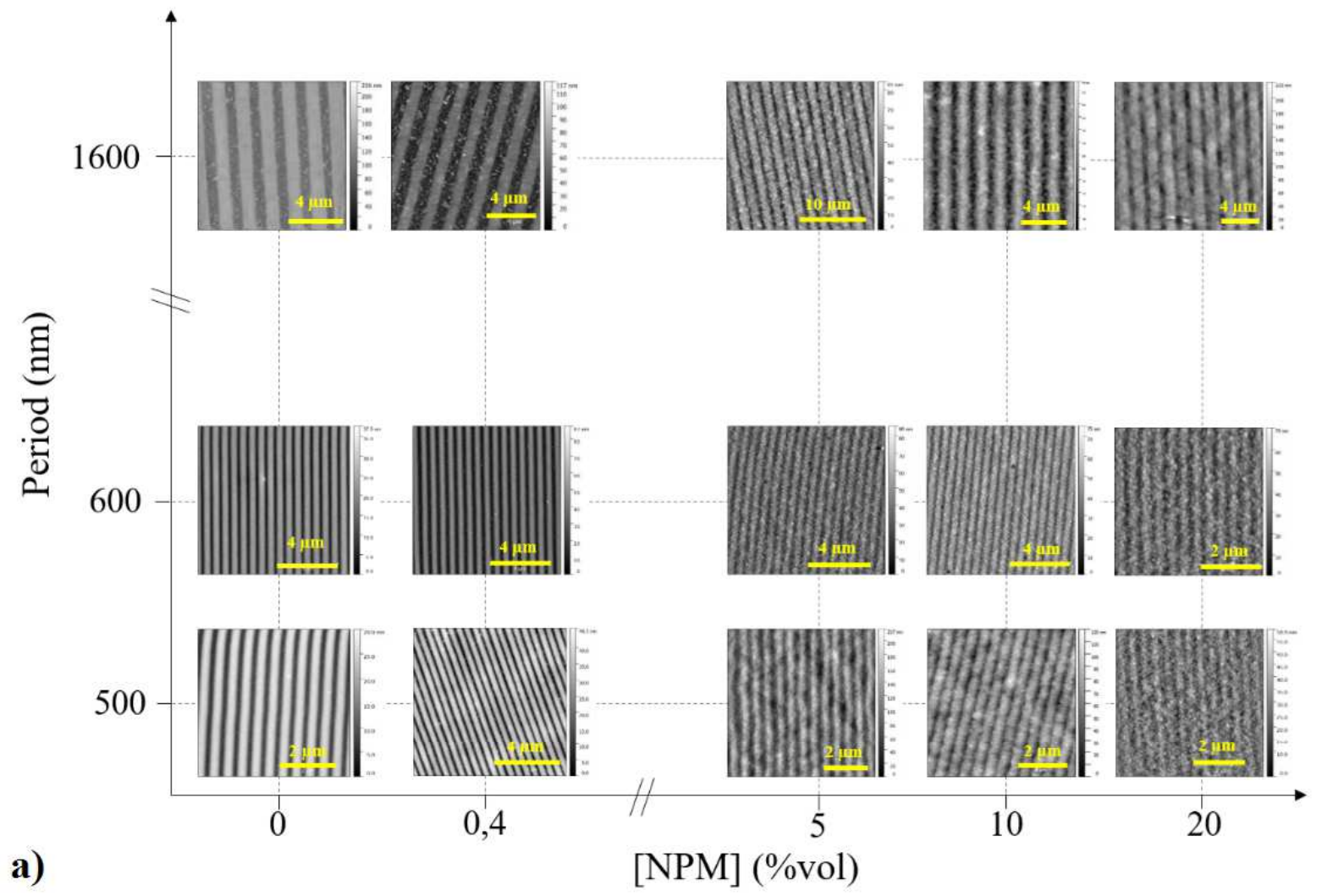

b)

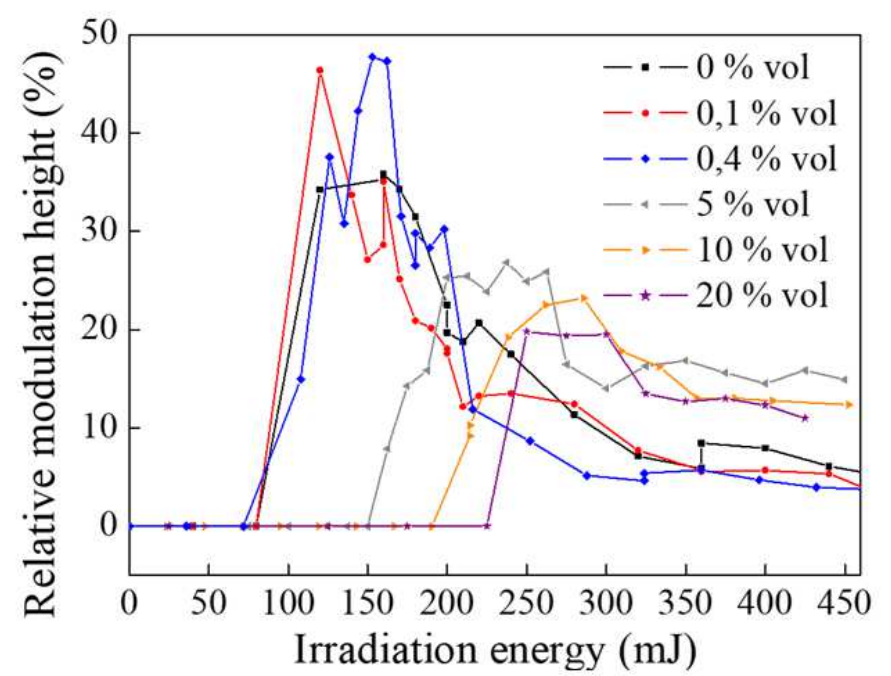

Figure 3. a) AFM topography images for structures with periods $1.6 \mu \mathrm{m}, 600 \mathrm{~nm}, 500 \mathrm{~nm}$, and for particle concentrations in the $0-20 \%$ vol range. b) Relative modulation height versus the irradiation energy and MNP concentration, for structures with the period of $600 \mathrm{~nm}$ and initial thickness of $8 \pm 5 \mathrm{~nm}$. 


\section{WILEY-VCH}

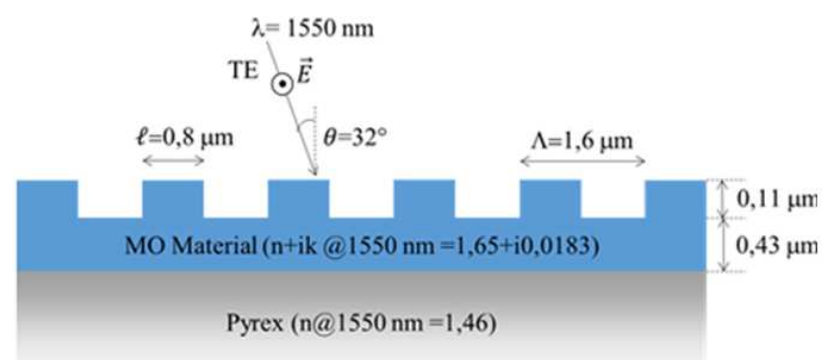

a)

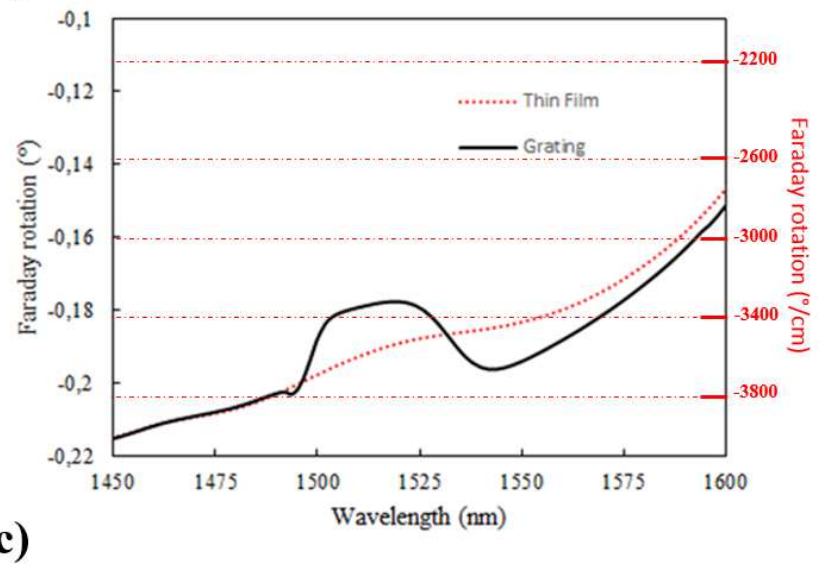

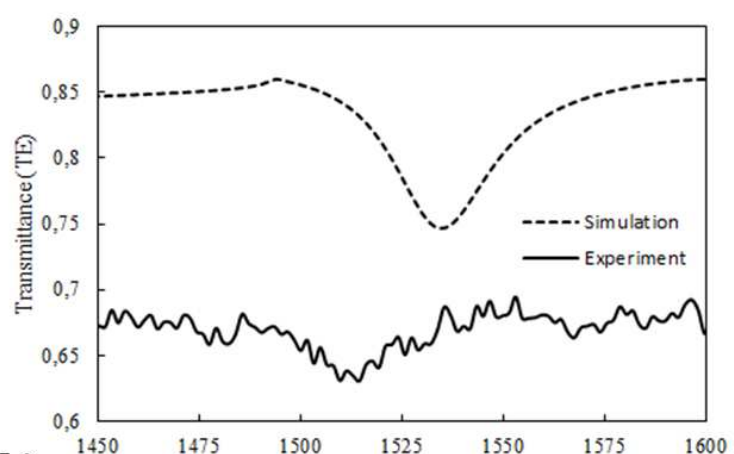

b)

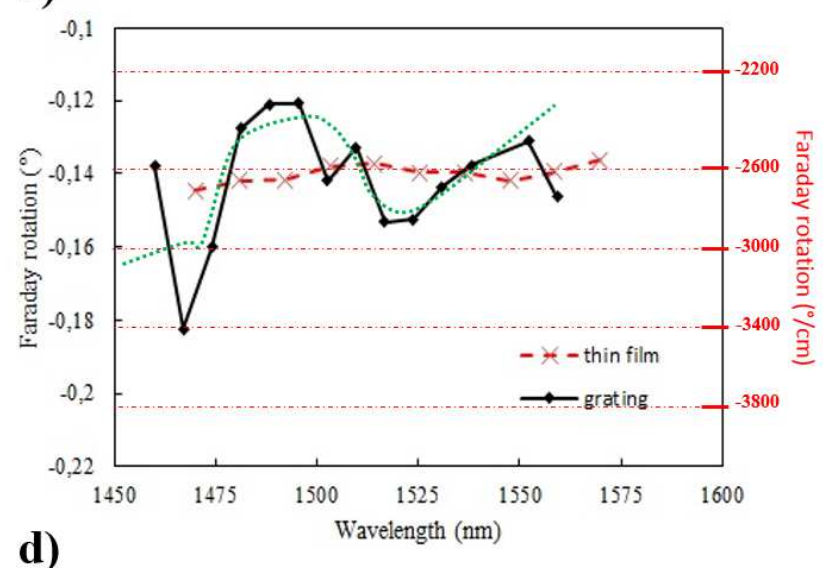

Figure 4. a) Schematic of the grating sample: period, $1.6 \mu \mathrm{m}$; depth, $110 \mathrm{~nm}$; sub-layer thickness, $430 \mathrm{~nm}$. b) RCWA simulation results (dotted curve) and measurement results (solid curve) of the TE transmittance. c) RCWA simulation results of the grating's Faraday rotation (solid curve) and the results for an equivalent thin film (red dashed curve). d) Measurement of the Faraday rotation of the photostructured grating and a non-structured zone (540-nm-thick film). The dotted green line is a guide for the eyes highlighting the MO activity of the grating. For c) and d) the scale in red on the right side corresponds to the Faraday rotation (in $\% \mathrm{~cm}$ ) of a 540-nm-thick film, for comparison with the data in Figure $2 \mathrm{~d}$. 


\section{WILEY-VCH}

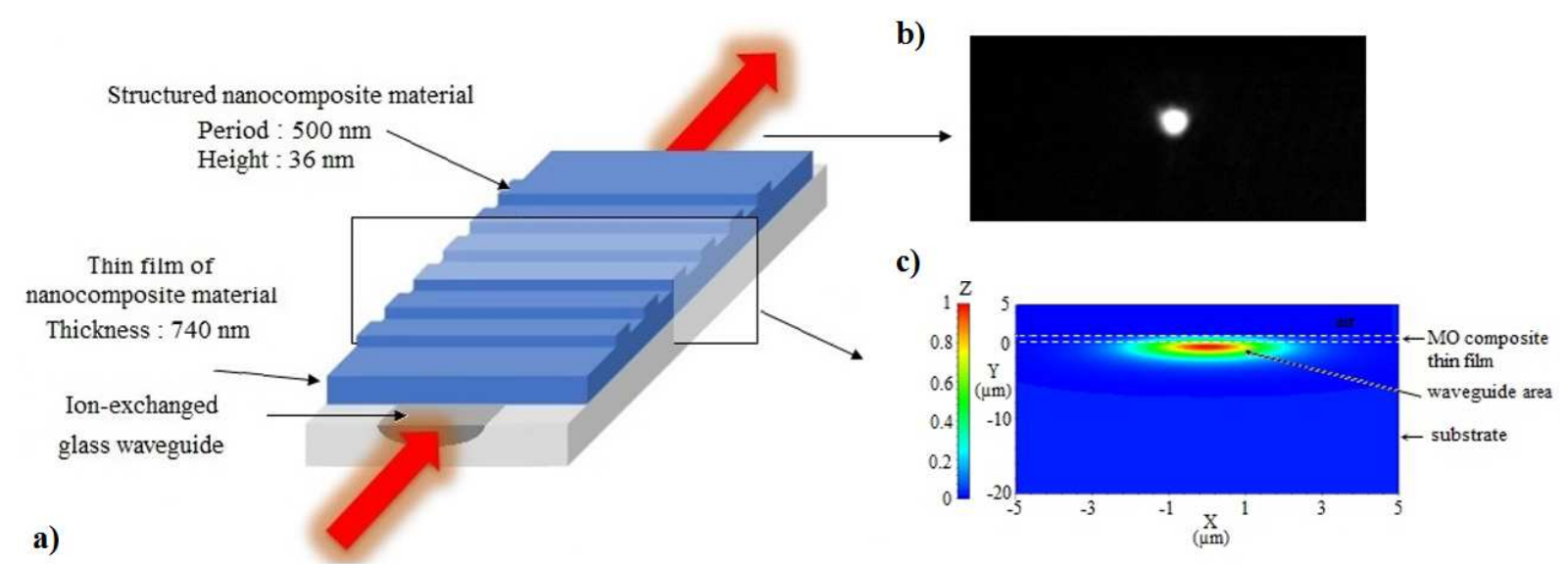

Figure 5. a) Schematic of the guiding sample prepared as a Bragg resonator on a waveguide. b) A photograph of the light beam exiting the guide. c) Simulation results of the guided mode field amplitude profile, performed using Optiwave BPM, for a 1- $\mu \mathrm{m}$-thick MO film on a waveguide. 


\section{WILEY-VCH}

\section{Table-of-Contents:}

This paper proposes a photolithography approach toward magneto-optical micro- and nanopatterns, based on hybrid photosensitive matrices doped with magnetic nanoparticles to concentrations as high as $20 \%$ vol. The Faraday rotation $(5300 \% \mathrm{~cm})$ is comparable with the best existing materials that nevertheless require much more involved processes. The photosensitive matrices allow patterned gratings with a wide range of periods ( $100 \mu \mathrm{m}$ to 500 $\mathrm{nm}$ ), and with suitable properties for optical applications at telecommunications wavelengths, in free space and guided optics.

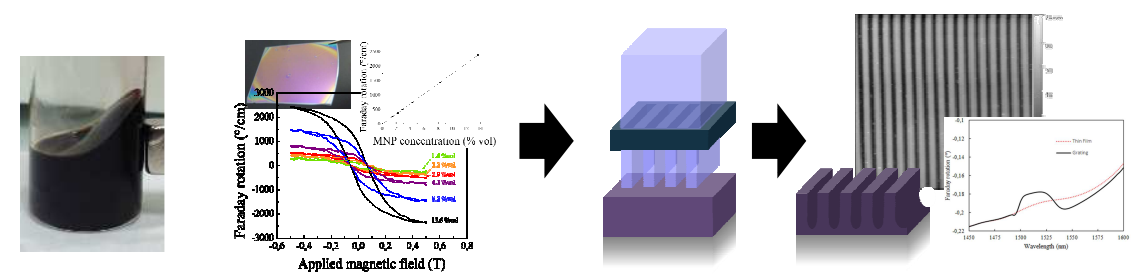

